\title{
Historia Natural y la Apropiación del Nuevo Mundo en la Ilustración española
}

L'histoire naturelle et l'appropriation du Nouveau Monde pendant l'Illustration espagnole

Natural History and the appropriation of the new world in the Spanish enlightenment

\section{Mauricio Nieto Olarte}

\section{OpenEdition}

Journals

\section{Edición electrónica}

URL: http://journals.openedition.org/bifea/6049

DOI: $10.4000 /$ bifea.6049

ISSN: 2076-5827

Editor

Institut Français d'Études Andines

Edición impresa

Fecha de publicación: 1 diciembre 2003

Paginación: 417-429

ISSN: 0303-7495

Referencia electrónica

Mauricio Nieto Olarte, « Historia Natural y la Apropiación del Nuevo Mundo en la llustración española », Bulletin de l'Institut français d'études andines [En línea], 32 (3) | 2003, Publicado el 08 diciembre 2003, consultado el 02 diciembre 2020. URL : http://journals.openedition.org/bifea/6049 ; DOI : https://doi.org/10.4000/bifea.6049

\section{c)}

Les contenus du Bulletin de l'Institut français d'études andines sont mis à disposition selon les termes de la licence Creative Commons Attribution - Pas d'Utilisation Commerciale - Pas de Modification 4.0 International. 


\title{
HISTORIA NATURAL Y LA APROPIACIÓN DEL NUEVO MUNDO EN LA ILUSTRACIÓN ESPAÑOLA*
}

\author{
Mauricio NIETO OLARTE***
}

\begin{abstract}
Resumen
Durante el siglo XVIII los viajes de exploración se convirtieron en el centro de intereses públicos, políticos y comerciales de las elites europeas. Ambiciosos proyectos de exploración a países lejanos fueron un esfuerzo común de los imperios europeos. La historia natural constituiría una forma de apropiación y jugaría un papel central en las políticas de Estado; el trabajo del naturalista clasificando y nombrando objetos naturales facilitaría el control no sólo de la naturaleza sino de otras culturas.
\end{abstract}

Palabras claves: Historia natural, Ilustración, exploración, Nuevo Mundo, apropiación.

\section{L'HISTOIRE NATURELLE ET L'APPROPRIATION DU NOUVEAU MONDE PENDANT L'ILLUSTRATION ESPAGNOLE}

\section{Résumé}

Au cours du XVIIIème siècle, les voyages d'exploration sont devenus le centre des intérêts publics, politiques et commerciaux des élites européennes. D'ambitieux projets d'exploration de pays lointains furent le résultat de l'effort commun des empires européens. L'histoire naturelle deviendra une forme d'appropriation et jouera un rôle central dans les politiques d'État ; le travail du naturaliste qui classifie et nomme les objets naturels facilitera le contrôle non seulement de la nature mais aussi des autres cultures.

Mots clés : Histoire naturelle, Illustration, exploration, Nouveau Monde, appropiation.

\section{NATURAL HISTORY AND THE APPROPRIATION OF THE NEW WORLD IN THE SPANISH ENLIGHTENMENT}

\section{Abstract}

During the eighteenth century, exploration trips became intense public interest for intellectual, commercial and political elites of Europe. Major exploration projects in distant

\footnotetext{
*Presentado en el XXI congreso de Historia de la Ciencia en Ciudad de México en julio de 2001; los argumentos centrales han sido desarrollados en detalle en Nieto, 2000.

${ }^{* *}$ Universidad de los Andes - Departamento de Historia. Carrera $1^{\text {a }} \mathrm{N}^{\circ} 18 \mathrm{~A}$ \# 0-33 Este Bogotá, Colombia. E-mail: mnieto@uniandes.edu.co
} 
countries became a common endeavor of European empires. Natural history, it will be shown, was a form of appropriation and a key function of state policy. The skills of naturalists would come understanding and eventual mastery not only of nature but also of other cultures.

Key words: Natural history, Enlightenment, Exploration, New World, Appropriation.

Durante el siglo XVIII los viajes de exploración se convirtieron en el centro de intereses públicos, políticos y comerciales de las elites europeas. Ambiciosos proyectos de exploración a países lejanos fueron un esfuerzo común de los imperios europeos. La historia natural constituiría una forma de apropiación y jugaría un papel central en las políticas de Estado; el trabajo del naturalista clasificando y nombrando objetos naturales facilitaría el control no sólo de la naturaleza sino de otras culturas.

Este es un período durante el cual los europeos sintieron que su poder sobre la naturaleza se incrementaba, pues no sólo habían logrado conquistar buena parte del globo terrestre, sino que también habían promulgado el descubrimiento de las leyes físicas que rigen el universo.

Desde la llegada de los europeos a América en el siglo XV, España tuvo bajo su control el más grande imperio colonial del mundo. Durante el reinado de Carlos III, fueron implementadas algunas reformas políticas que buscaban optimizar la explotación de las colonias estimulando la exploración científica de América. Siguiendo los parámetros de la Ilustración Francesa, el gobierno español basó sus políticas en el supuesto de que la adquisición y aplicación de conocimientos científicos incrementaría su poder político y económico. La clave de la prosperidad económica del imperio español parecía yacer en una explotación más eficiente de la riqueza natural de sus colonias.

Durante la segunda mitad del siglo XVIII, el gobierno español diseñó y llevó a cabo un número de ambiciosas expediciones a cargo de botánicos que debían investigar los posibles usos medicinales y comerciales de la vegetación tropical. Los proyectos de exploración estaban dirigidos por médicos y patrocinados por instituciones médicas. En España, más que en ninguna nación europea, la familiaridad con plantas medicinales y la promoción de una industria farmacéutica española se convirtieron en compromisos centrales del Estado. La vieja relación entre el reino vegetal y la medicina le permitió a la botánica jugar un papel vital en las políticas económicas imperiales.

Con el apoyo de la Corona, los exploradores permanecieron en América por varios años recolectando numerosas especies y llevando a Europa grandes colecciones de plantas disecadas, ilustraciones botánicas, muestras y descripciones de especimenes considerados útiles. Los informes y diarios de diferentes exploradores españoles contienen cientos de referencias de plantas medicinales, algunas de las cuales tuvieron un impacto considerable sobre la industria farmacéutica europea. El caso de la quina es, entre muchos otros, un ejemplo notable de la intersección entre factores médicos, científicos y comerciales. 


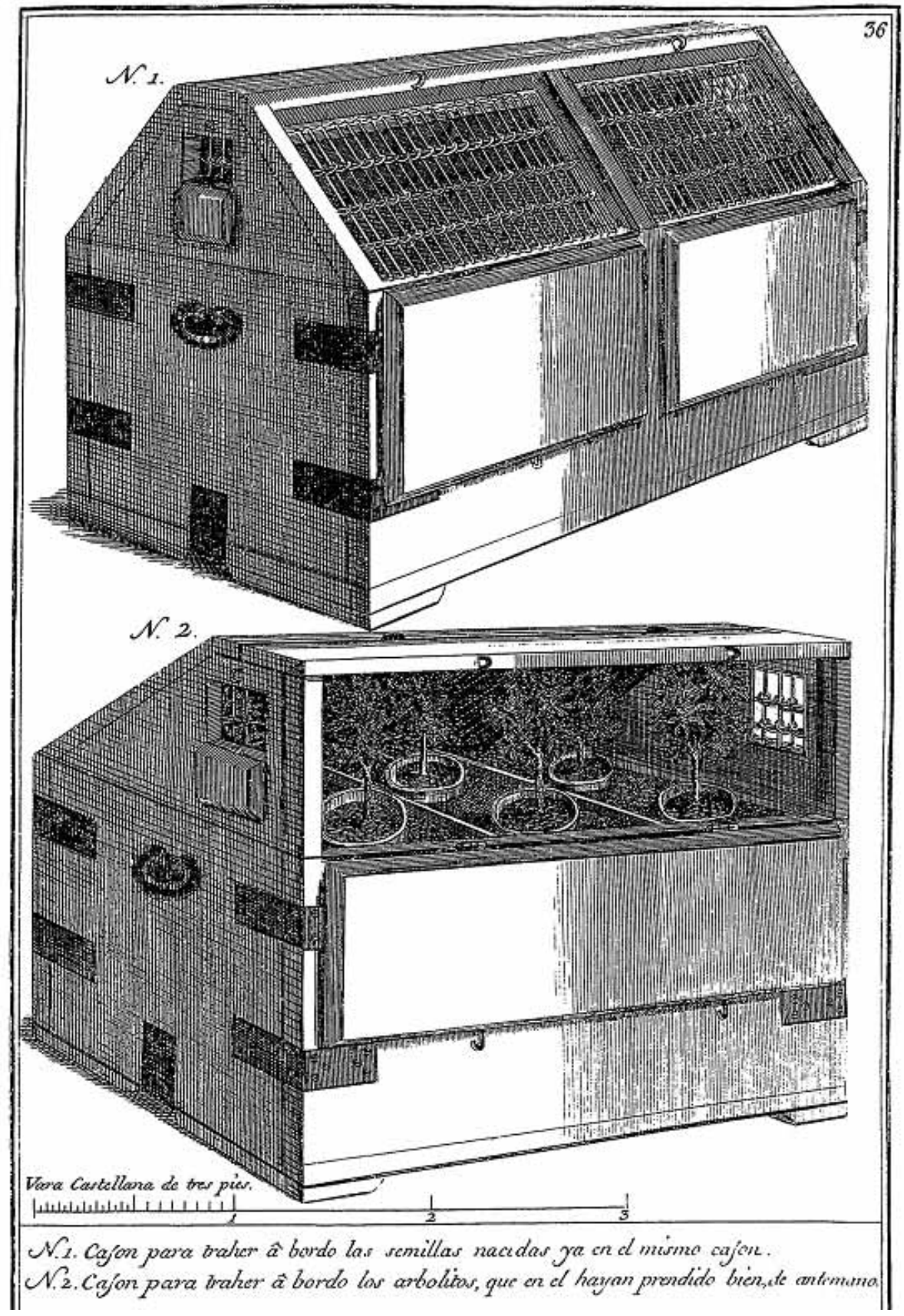

Fig. 1 - Cajón para transportar semillas en germinación y árboles pequeños. Instrucción sobre el modo más seguro y económico de transportar plantas vivas de Casimiro Gómez Ortega (Madrid 1779). 
Algunas de las discusiones contemporáneas en historia y sociología de la ciencia nos ofrecen herramientas para la elaboración de un nuevo y más crítico estudio de los viajes de exploración en la historia política de Europa y sus colonias.

El reconocimiento del carácter social de las prácticas científicas nos ha permitido explicar como la ciencia del siglo XVIII en América, la historia natural y la medicina principalmente, hacen parte de intereses políticos, económicos y religiosos; que las políticas económicas coloniales estimularon el desarrollo de la farmacia y la taxonomía vegetal, y que dichas prácticas constituyen importantes formas de control tanto de la naturaleza como de la sociedad.

El proyecto de un inventario del mundo no se puede separar de la conquista y el control de buena parte del planeta por parte de las naciones más fuertes de Europa. La historia natural es un medio para construir una naturaleza doméstica y una humanidad colonizada. Por lo tanto, la historial natural y la política deben ser consideradas expresiones de la misma estructura de poder. Sería un serio error pretender imaginar que el conocimiento de la naturaleza no es parte de un orden social y es importante que tratemos de evadir contraposiciones entre nociones como "sociedad", "poder", "política”, por una parte, y “conocimiento", por otra (Barnes, 1988; Rouse, 1987).

Conocimiento, descubrimiento, apropiación y poder: estos son todos conceptos claves para entender el papel del estudio de la naturaleza en la Ilustración europea. El "poder", como ha sugerido Barnes (1988), puede ser entendido como "posesión". Posesión de territorio, productos comerciales, armas o tecnología. La idea de "descubrimiento", como veremos, implica un acto de apropiación. "Descubrimiento" ha sido tradicionalmente entendido como encontrar algo que existía pero que nadie había visto. Sin embargo, para que cualquier objeto natural pueda ser "visto" o "descubierto", debe ser transformado en algo familiar conforme a un sistema ya conocido, y de cierta manera todo objeto "descubierto" tiene que haber pasado por un proceso de construcción (Brannigan, 1981).

Las habilidades de los naturalistas europeos para clasificar la naturaleza dándole nombres a plantas y animales y sus técnicas de representación son instrumentos de apropiación. Quién por primera vez reconoce un lugar, una planta o una medicina proclama su derecho de posesión. Dichos procesos de apropiación sólo son posibles dentro de redes de cooperación que hagan posible la movilización, clasificación, codificación, exhibición e inclusive la venta de los objetos de estudio.

Para entender el desarrollo de estos procesos debemos explicar como se construye una red de traducción amplia y compleja que hace posible que ciertas personas o grupos sociales asuman el papel de portavoces del nuevo orden de la naturaleza y la sociedad. Estas redes, tal y como lo propone Michel Callon (1986), las componen actores de muy diversa índole. Organismos sociales, actores humanos con habilidades de producir artefactos técnicos, los artefactos mismos, ilustraciones, textos, objetos naturales, la geografía americana, la vegetación y desde luego los nativos americanos.

“Traducir es desplazar...”, afirma Callon (1986), "Traducir es también expresar en un lenguaje propio lo que otros dicen o hacen, es hacer de uno mismo el portavoz...". Toda traducción implica remover algo de una persona o cultura, llevar, transportar 
algo de un lugar a otro. El resultado, diría Callon, "es una situación en la cual ciertas personas controlan a otras".

La historia natural es una práctica que se construye sobre redes en la cual las muestras del mundo natural, los objetos de estudio del naturalista, tienen que ser movilizados desde los lugares más remotos a los principales centros culturales; la naturaleza, para ser dominada debe convertirse en cultura. Para que esto sea posible es necesario desarrollar técnicas de preservación de los objetos, vivos o disecados, o técnicas de representación que permita su apropiación "virtual". Son estas redes las que generan la necesidad de sistemas "universales" de códigos y reglas bien definidos para poder acumular información en centros como París, Londres o Madrid.

El proyecto totalizador de catalogar el mundo incluye el difícil trabajo de extraer objetos naturales de sus medios particulares. Para ser transportables los objetos debían ser estables y al mismo tiempo móviles. Medios efectivos para la clasificación, transporte, representación y documentación de los especímenes eran necesarios para evitar el deterioro de las plantas en travesías intercontinentales.

Esta acumulación y apropiación de objetos naturales fue posible porque fueron solucionados problemas de movilización. La tarea de los naturalistas era convertir y transformar lo inconmensurable en conmensurable, hacer familiar lo desconocido, crear un vínculo y, en últimas, poseer lo extraño. (Pagden, 1993; Greenblatt, 1991)

Instituciones como el Jardin du Roi, más tarde, Jardin des Plantes en París, Kew Gardens en Londres o el Real Jardín Botánico en Madrid, se convirtieron en estancos del conocimiento, lugares claves en Europa donde la información recogida en viajes de exploración era recreada de tal manera que se celebra y reconoce la expansión europea. La descontextualización de objetos y su re-acomodación en estos centros requiere de una serie de técnicas como disecar animales, secar plantas, clasificar especímenes, transportarlos, hacer ilustraciones y dibujos de especies animales y paisajes. Estas técnicas le daban a la naturaleza un nuevo contexto y un nuevo lenguaje que hizo a la naturaleza descifrable para los europeos.

Las instituciones científicas europeas como jardines botánicos o museos de historia natural, Kew Gardens en Londres, Jardin des Plantes en París, o el Real Jardín Botánico de Madrid fueron grandes casas de intercambio que buscaban hacer que cada nación fuera independiente y autosuficiente de los otros poderes imperiales.

Tales instituciones conformaron redes de colaboradores a través de todo el mundo; fueron lugares donde un pequeño grupo de naturalistas estaban en capacidad de comparar plantas y animales en una escala completamente distinta de la de los nativos. Los botánicos que se encontraban en tales centros adquieren mayor capacidad de control de la naturaleza que los mismos habitantes de los lugares explorados. De hecho se familiarizaron con más especímenes que cualquier otro ser humano a medida que más y más objetos provenientes de lugares lejanos eran domesticados.

Los viajeros y naturalistas españoles actuaron como agentes tanto del Estado como de Dios y sus descubrimientos o actos de apropiación, aunque proclamados individualmente, son presentados en nombre del Rey y con una fuerte justificación religiosa. Los logros de las expediciones fueron muestra de soberanía y los jardines botánicos y los museos de historia natural se convirtieron en galerías públicas donde 
los imperios europeos podían exhibir tanto su poder como la obra del Creador. Durante el siglo XVIII cualquier centro cultural que se respetara debería estar en capacidad de mostrar colecciones de especimenes naturales, plantas, animales o minerales de lugares remotos. Museos nacionales de historia natural fueron establecidos en Londres (1753) y París (1745); jardines botánicos en Viena (1751), Madrid (1755), Lyon y Nancy (1758), Cambridge (1762) y Versalles (1765). Todas estas instituciones se convirtieron en importantes símbolos de poder. Coleccionistas aristócratas empezaron a satisfacer sus intereses y capacidades adquisitivas no sólo con obras de arte sino también con fósiles, animales disecados, conchas, minerales y mariposas, muchas veces adquiridas a precios elevados. Como señala Peter Bowler (1992: 14):

"el mundo natural fue incorporado en el mundo de la propiedad."

Es importante reconocer el carácter institucional y político de la historia natural; ver como la credibilidad de los naturalistas depende en gran medida del poder de la institución en que trabajan y de la posición social que les otorga el oficio de la historia natural. La popularidad de la historia natural tiene que ver con una serie de prácticas sociales y proyectos políticos alrededor de colecciones, gabinetes, exhibiciones y jardines, que les dan estatus a sus propietarios. La historia natural era una disciplina cuyos practicantes dependían del patrocinio de la aristocracia o del Estado ya que solamente los más ricos de la sociedad europea estaban en capacidad de adquirir objetos raros y acumular colecciones de objetos exóticos.

Los centros de producción de los últimos conocimientos o avances en historia natural en el siglo XVIII son los gabinetes y los jardines de los aristócratas más poderosos o de la nobleza.

Los naturalistas del siglo XVIII hacen del estudio de la naturaleza un elemento esencial de una educación civilizada. La habilidad de comentar una colección es una muestra de educación. Los naturalistas tienen una importante función social en la medida en que hacen de la naturaleza, de lo salvaje algo ordenado y placentero. La naturaleza bruta debe ser organizada por el hombre.

Los museos y jardines son expresiones y símbolos de poder de Europa sobre lo salvaje y del hombre sobre las bestias.

Los intereses europeos (españoles) antes del siglo XVIII se habían concentrado en productos como oro y plata fácilmente transportados, almacenados e intercambiados. Las plantas son delicadas y se dañan con facilidad. Su apropiación requirió de técnicas más sofisticadas. En ocasiones no podían ser transportadas, pero podían ser reemplazadas por dibujos y nombres.

Los nombres nativos parecen perder toda importancia y repetidamente los expedicionarios los señalan como nombres ilegítimos. Para Antonio de Ulloa:

"el lenguaje Quechua de los Incas se aproxima más al lenguaje de los niños".

Al parecer, los nativos no comprendían las palabras y los conocimientos propios de cualquier "sociedad civilizada" como "Dios", "virginidad" o "inmaculada concepción". Los indígenas americanos tenían innumerables nombres para plantas pero no una única palabra que se pudiera traducir como “árbol” (Pagden, 1993: 132). Culturas 


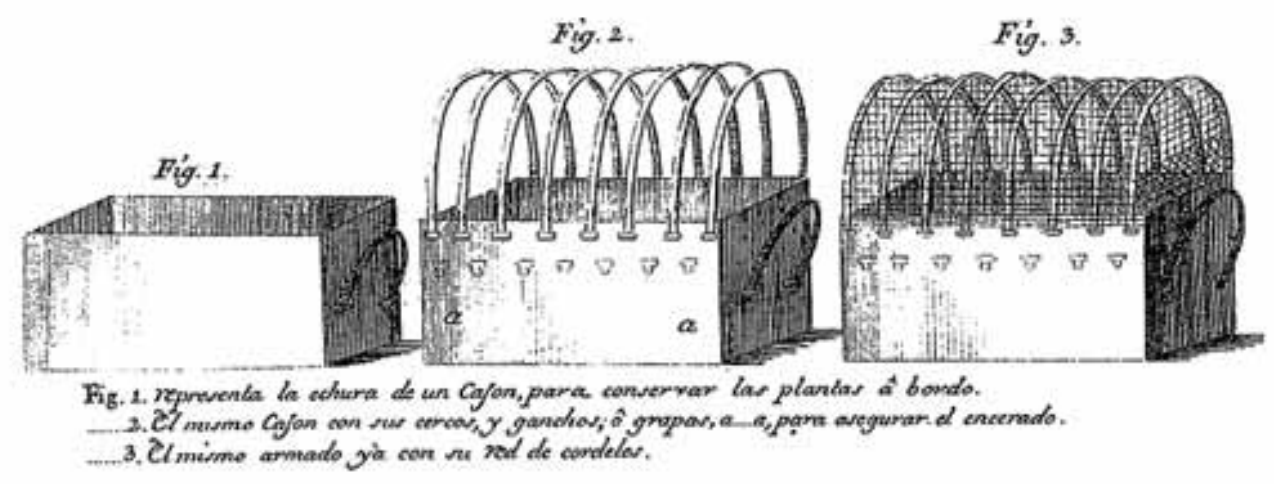

\section{ARTICULO PRIMERO.}

INSTRUCCION PARA ARRANCAR CON SU CESPED LOS ARBOLITOS, ARBUSTOS, MATAS, $x$ DEMAS PLANTAS PERENNES, $T$ TRANSPLANTARLAS, NO SOLO DE UNA PROVINCIA A' OTRA, SINO TAMBIEN POR TIERRA $r$ MAR $A$ LOS: PAISES MAS DIST ANTES.

I ara arrancar del modo mas conveniente las plantas y arbolitos, se deberá llevar á prevencion un pico con azada, y una pala de cavar. Con el primer instrumento se abrirá una zangilla, ó reguera circular al rededor de la planta que se vaya á sacar de tierra; é introduciendo luego la azada por

Fig. 2 - Cajón para transportar plantas a bordo. Instrucción sobre el modo más seguro y económico de transportar plantas vivas de Casimiro Gómez Ortega

(Madrid 1779). 
cuya supervivencia dependía del conocimiento y uso de la vegetación circundante, reconocían numerosas plantas de utilidad, sabían cuáles eran sus usos, y le habían dado nombres descriptivos. Sin embargo, es obvio que no compartían con los naturalistas conceptos como especie, género o clase.

Para conquistar plantas extrañas, el europeo se debe deshacer de contingencias locales y fabricar tipos ideales conformes con el sistema de clasificación europeo.

También debemos explicar cómo el proceso de traducción de lo local a lo global se lleva a cabo. El conocimiento que los exploradores buscaban no sólo pertenecía a continentes lejanos sino también a sus habitantes.

En el caso de la búsqueda y "descubrimiento" de plantas útiles, en particular de uso medicinal, es evidente que los exploradores europeos no estaban en capacidad de probar todas las especies que encontraban, no les era posible hacer experimentos ni análisis químicos, de manera que lo normal es que su primera fuente de información y los primeros indicios sobre la utilidad de una planta provenían de los usos y conocimientos locales. Conocimientos que para ganar credibilidad en el mundo de la ciencia ilustrada debieron ser traducidos e incorporados en el lenguaje y las doctrinas de la ciencia europea.

Como lo sugiere Pratt (1992) la sistematización de la naturaleza fue un proyecto europeo que siguió la circunnavegación del mundo cuando los cartógrafos se concentraban en las líneas costeras de los nuevos continentes. Este nuevo proyecto de "conciencia planetaria" tuvo como foco la exploración del interior de los continentes y fue acompañado por una más fuerte y reforzada imposición de unos valores y una cultura particular.

La apropiación no se limitó entonces a las líneas costeras, sino que incluía cada objeto del planeta: plantas, aves, peces, insectos, minerales y gente. La colonización europea no sólo supuso la gradual eliminación de las prácticas medicas locales, sino que el orden de la naturaleza propio de las distintas culturas también fue negado.

Este proyecto global de ordenar la creación de Dios necesariamente implicó la reincorporación de la naturaleza en un patrón de unidad y orden euro céntrico y cristiano. La visión europeo cristiana de la naturaleza nunca abandonó la idea de que el fin de la creación y de cada uno de sus objetos era el beneficio del hombre. Siempre hubo, como lo ejemplifica la obra de Linneo, una visión teleológica y funcional de la naturaleza, como si esta hubiese sido creada para el hombre, y en particular para el hombre europeo.

La taxonomía es fundamental para la diseminación del poder; es una ciencia que delimita y demarca objetos, organiza dominios y establece fines. Como explica David Mackay (1996):

"en la medida en que los recolectores penetran otras culturas se transforman en agentes del imperio en un sentido más profundo. Sus inventarios, clasificaciones y movilizaciones eran la vanguardia y los instrumentos de un orden europeo que se imponía en todo el mundo."

Ordenar el mundo natural es una actividad inseparable del compromiso de controlar e imponer un orden sobre otras culturas. 
No tendríamos una visión completa del papel de las expediciones y de la ciencia colonial sin explicar de que manera la historia natural también comienza a practicarse dentro de los continentes y en manos ya no sólo de viajeros sino de habitantes nativos; y que estos proyectos europeos de exploración y de implantación de la ciencia occidental no hubieran sido posibles sin la colaboración de una elite americana interesada en adoptar los métodos y las ambiciones de la ciencia europea. (Lafuente \& Lopez-Ocón, 1996)

La historia natural, la medicina y la astronomía pronto se convertirían en la profesión de hombres no-europeos. Debe tenerse en cuenta que Mutis y muchos otros científicos europeos vivieron buena parte de sus vidas o incluso terminaron sus vidas en América. No sólo entrenaron en medicina o historia natural a algunos americanos, sino que fundaron instituciones como jardines botánicos, museos, observatorios astronómicos y cátedras universitarias. Una vez estas instituciones fueron creadas y contaron con naturalistas entrenados, se convirtieron en símbolos de poder local, en satélites de un gran proyecto de ordenamiento global bajo el control de una nueva elite de hombres nacidos en América pero de sangre europea.

Alrededor de José Celestino Mutis y la Real Expedición Botánica, encontramos una elite de americanos que se convirtieron en practicantes y promotores de actividades científicas. Los miembros y colaboradores de la expedición constituyeron un grupo de criollos ilustrados en cuyas manos la historia natural, la medicina, la geografía y la astronomía se convierten en la expresión de sus propios intereses políticos.

El proceso de apropiación que identifica la exploración europea del Nuevo Mundo comienza a echar raíces a miles de kilómetros de Madrid; siendo este un proceso sobre el cual la Corona perdería control directo. El proyecto de apropiación ya no era una tarea de viajeros europeos y pasó a manos de la elite local. Este grupo de americanos - o mejor de europeos nacidos en América - compartía con los exploradores la educación, el propósito y el deseo de dominar la naturaleza pero sus vínculos con las instituciones y la ciencia europea son menos sólidos y su papel como agentes y beneficiarios del imperio menos claro. Existe una estrecha relación entre los hombres de ciencia, su identidad social y el poder que prácticas como la historia natural otorgan. Muchos de ellos se convirtieron en personajes influyentes con altos puestos oficiales y un considerable control político antes, durante y después de las guerras de independencia. Sin excepción, todos eran de ascendencia española y tuvieron acceso a educación europea. Algunos de ellos fueron ejecutados por las autoridades españolas debido a sus vínculos con la independencia y rápidamente fueron convertidos en modelos de orgullo nacional, en héroes de las clases dominantes de las nuevas naciones americanas.

El caso de Francisco José de Caldas en la Nueva Granada es un claro ejemplo de cómo la elite criolla encuentra en las preocupaciones y actividades propias de la Ilustración europea un efectivo mecanismo de reconocimiento social y en la ciencia un espacio para el ejercicio de la política.

Tanto para los líderes de las nuevas naciones como para las autoridades españolas, la adquisición de conocimiento y las prácticas científicas constituyeron rutas esenciales para proclamar soberanía sobre el continente americano. Pascual Enrile, el general a cargo de la armada española para recobrar la Nueva Granada le escribió al secretario de Estado: 


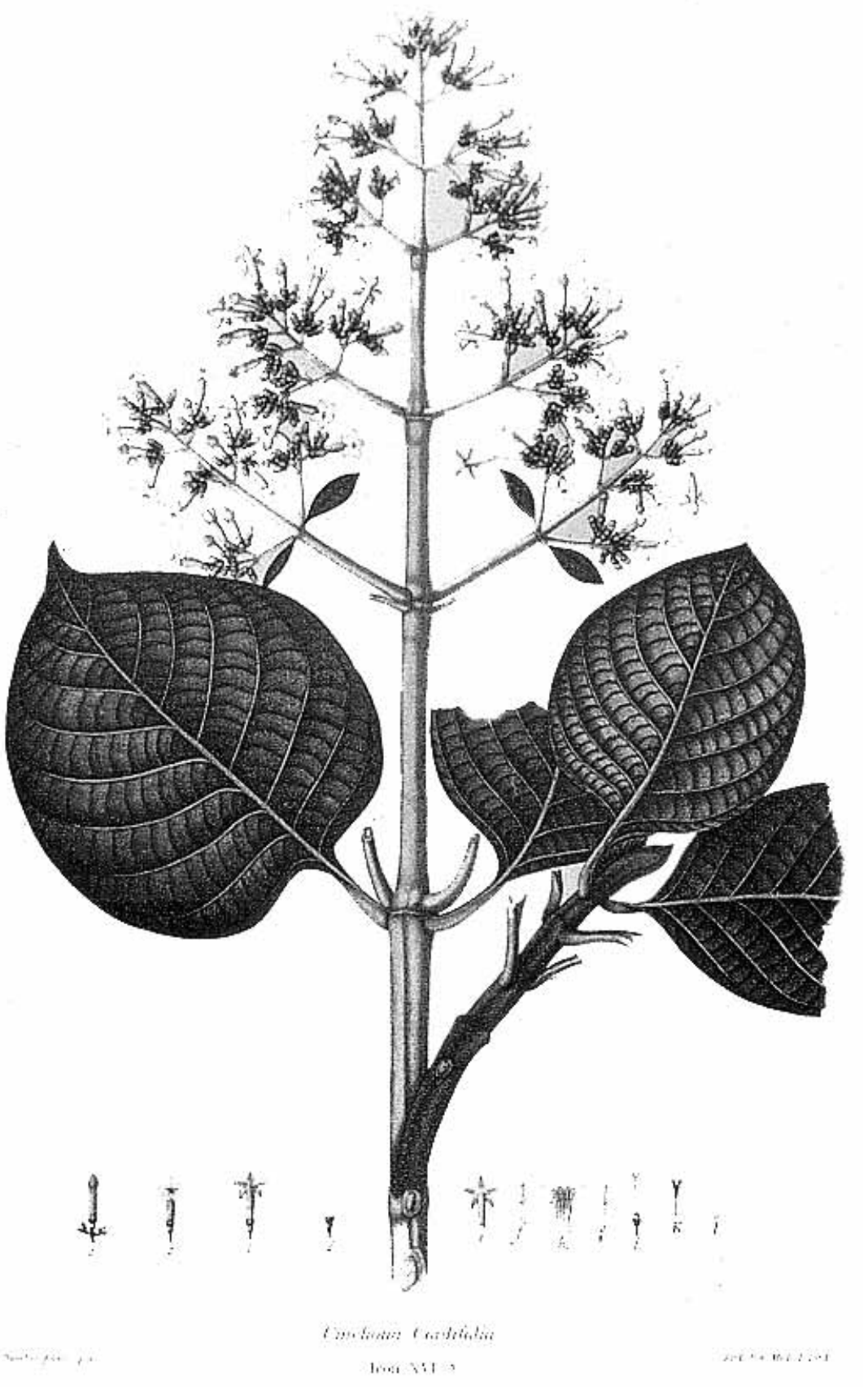

Fig. 3 - Cinchona cordiflolia. Flora de la real expedición Botánica del Nuevo Reino de Granada. 
"Los insurgentes se ocuparon mucho de la geografía del país y después quisieron enterarse de la topografía. Sacaron de los archivos del Virrey, Audiencia, monasterios y cuanto había lo vendieron a los encargados de la Botánica y teniendo a la vista las muchas observaciones de Caldas, las de Humboldt, las de los marinos y el mapa de Talledo, emprendieron la grande obra de un mapa del Virreinato" (Hernández de Alba, 1986: $353)$.

Una de las tareas centrales de la armada española era re-poseer los materiales, libros e instrumentos de la casa de la Expedición Botánica y del observatorio. En 1818 la Gaceta de Madrid publicó una versión del "éxito" de la armada española, no solamente en restablecer el orden en varios lugares de América sino en recobrar los numerosos objetos de historia natural del señor Mutis.

Los materiales fueron enviados a Madrid, al palacio del Rey, donde el mismo Rey oficialmente tomaría posesión. Ordenó que los especímenes minerales y de zoología fueran guardados en el Museo de Historia Natural, y el herbario y las ilustraciones botánicas en el Real Jardín Botánico. Dispuso además la pronta publicación de todos los materiales relacionados con La Flora de la Nueva Granada. Los libros e instrumentos del observatorio fueron confiscados y enviados a Madrid.

Por su parte, los criollos también hicieron todos los esfuerzos por la reapropiación del continente buscando el reconocimiento como legítimos portavoces del orden natural. A través del siglo XIX la historia de la ciencia en Colombia se puede ver como un continuo esfuerzo por continuar las tareas de la Ilustración. La geografía, la historia natural, la mineralogía entre otros, serán compromisos de las agendas políticas de los nuevos líderes en América. Ya para 1811, la constitución de Cundinamarca incluía el establecimiento de una sociedad patriótica en la cual la política europea de apropiación se transforma en una política local.

"Deberá establecerse cuanto antes en la capital una Sociedad Patriótica, así para promover y fomentar estos establecimientos en ella y en toda la Provincia, como para hacer otro tanto en razón de los ramos de ciencias, agricultura, industria, oficios, fábricas, artes, comercio, etc... Entre los demás establecimientos, se tendrá presente el de la Expedición Botánica, para extenderlo, además de los trabajos en que hasta ahora se hubiese empleado, a la enseñanza de las ciencias naturales, bajo la inspección de la Sociedad Patriótica." (Hernández de Alba, 1986: 216)

Durante las primeras décadas de la República (1822-1850) el gobierno haría todo tipo de esfuerzos por crear vínculos y ganar reconocimiento internacional. En 1823, el gobierno de Colombia aprobó la contratación de 5 naturalistas franceses, por medio de Zea, para la fundación de un Museo Histórico Natural y una Escuela de Minería en Santafé (Safford, 1985). En este proyecto el gobierno termina pagando altos salarios a técnicos extranjeros e importando instrumentos y materiales para promover una investigación que parecía beneficiar más a las mismas instituciones científicas europeas que resolver las necesidades de la nueva nación. También es significativo que la primera gran empresa científica financiada por el gobierno nacional fue la "Comisión Corográfica" (1850-1859) para una investigación sistemática y elaboración de mapas del territorio nacional. 
No pretendo explicar o cuestionar el proceso de independencia de las colonias españolas; los numerosos factores políticos, económicos y sociales relacionados con el nacimiento de las nuevas naciones americanas están por fuera de los propósitos de esta presentación. Tampoco tendría sentido desconocer los méritos de personajes como Mutis, Caldas, Zea o Lozano. Se trata más bien de señalar que la cultura y la naturaleza, lo natural y lo social no son facetas de la realidad que se puedan separar. Lo interesante es poder entender cómo una serie de prácticas científicas y su proceso de diseminación constituyeron importantes formas de ejercer poder.

Frecuentemente los historiadores han señalado relaciones entre los intereses del Estado y los fines de la exploración científica. Esos análisis pueden ser de gran utilidad, pero el punto aquí es comprender al conocimiento como una forma de poder y apreciar que la diseminación de disciplinas como la historia natural, la taxonomía y la medicina es la diseminación del poder. Desde esta perspectiva, encontramos razones para argumentar que después de las guerras de la independencia - comúnmente relacionadas con los ideales progresistas de la Ilustración - la estructura de poder permaneció, en gran medida, sin modificación. La soberanía de la Corona española no se reconocía formalmente, pero las colonias ya habían adoptado o estaban adoptando formas de dependencia aún más profundas, la lengua, la religión y la ciencia.

Desde entonces la historia de la ciencia en Colombia ha estado marcada por un continuo esfuerzo local para obtener el reconocimiento internacional lo cual en parte explica que aún hoy en día no sea posible reconocer una comunidad científica nacional fuerte con un verdadero impacto sobre las necesidades de la sociedad colombiana.

\section{Referencias citadas}

BARNES, B., 1988 - The nature of power, 205p.; Cambridge: Polity Press.

BOWLER, P., 1992 - The Fontana History of environmental sciences, 464p.; Londres: Fontana Press.

BRANNIGAN, A., 1981 - The Social Basis of Scientific Discoveries, 212p.; Cambridge: Cambridge University Press.

CALLON, M., 1986 - Some elements of a sociology of translation: domestication of the scallops and the fishermen of St Brieuc Bay. In: Power, action and belief (John Law Ed.): 196233; Londres: Routledge \& Kegan Paul.

HERNÁNDEZ DE ALBA, G. (Ed.), 1986 - Historia documental de la Real Expedición Botánica del Nuevo reino de granada después de la muerte de su director Don José Celestino Mutis, 1808-1952, 489p.; Bogotá: Instituto Colombiano de Cultura Hispánica.

LAFUENTE, A., ELENA, A. \& ORTEGA, M. L.,(Ed.), 1993 - Mundialización de la ciencia y cultura nacional, 749p.; Madrid: Doce Calles.

LAFUNTE, A. \& LÓPEZ-OCÓN, L., 1996 - Tradiciones científicas y expediciones ilustradas en la América hispana del siglo XVII. In: Historia social de las ciencias en América latina (Saldaña, Juan José Coord.): 247-281; Mexico. 
MACKAY, D., 1996 - Agents of empire: the Banksian collectors and evaluation of new lands. In: Visions of Empire: voyages, Botany and Representation of Nature (Miller, David \& Reil, Peter Ed.); Cambridge: Cambridge University Press.

NIETO, M., 2000 - Remedios para el imperio: Historia Natural y la apropiación del Nuevo Mundo, 279p.; Bogotá: Instituto Colombiano de Antropología e Historia.

PAGDEN, A., 1993 - European encounters with the New World: from Renaissance to Romanticism, 216p.; New Haven: Yale University Press.

PRATT, M. L., 1992 - Imperial Eyes, Travel, writing and transculturation, 257p.; Londres: Routledge.

PUERTO-SARMIENTO, J., 1988 - La ilusión quebrada: Botánica, sanidad y política científica en la España ilustrada, 315p.; Madrid: Serbal CSIC.

PUIG-SAMPER, M. A., 1991- Las expediciones científicas durante el siglo XVIII, Madrid: Akal.

ROUSE, J., 1987 - Knowledge and power: toward a political philosophy of science, 304p.; Londres: Cornell University Press.

SAFFORD, F., 1985 - Acerca de la incorporación de las ciencias naturales en la periferia: el caso de Colombia en el siglo XIX. Quipu, 2: 423-435. 